\title{
MicroRNA-758 inhibits malignant progression of retinoblastoma by directly targeting PAX6
}

\author{
JUN LI and XINYING YOU \\ Department of Ophthalmology, Liaocheng People's Hospital, Liaocheng, Shandong 252000, P.R. China
}

Received January 25, 2018; Accepted May 31, 2018

DOI: $10.3892 /$ or.2018.6563

\begin{abstract}
Accumulated studies have highlighted that the dysregulation of microRNAs (miRNAs) in retinoblastoma (RB) is a leading cause for tumourigenesis and tumour development. Therefore, the elucidation of the expression, functional roles and underlying mechanisms of miRNAs in RB will help the development of promising therapeutic methods to improve the prognosis of RB patients. The aim of this study was to detect miRNA-758 (miR-758) expression in RB tissues and cell lines, and to determine the effects and underlying mechanisms of miR-758 on RB progression. The results demonstrated that miR-758 was downregulated in both $\mathrm{RB}$ tissues and cell lines. In vitro functional experiments revealed that upregulation of miR-758 inhibited cell proliferation, migration and invasion, and induced apoptosis in RB. In addition, paired box protein 6 (PAX6) was a direct target gene of miR-758 in RB. Furthermore, PAX6 was upregulated in RB tissues, and this upregulation was inversely associated with the expression level of miR-758. In addition, PAX6 reintroduction abrogated the tumour-suppressive effects of miR-758 overexpression on RB cell proliferation, migration, invasion and apoptosis. Furthermore, miR-758 overexpression inactivated the PI3K/Akt pathway in RB cells by inhibiting PAX6. In conclusion, our current study provided sufficient evidence to demonstrate that miR-758 inhibits the progression of RB by directly targeting PAX6 and regulating the PI3K/Akt pathway, thereby suggesting that this miRNA may be developed as a therapeutic target for treating patients with RB.
\end{abstract}

\section{Introduction}

Retinoblastoma (RB), the most prevalent primary intraocular malignancy, is initiated from immature cells in the retina (1). RB typically arises in infancy and childhood and accounts

Correspondence to: Professor Xinying You, Department of Ophthalmology, Liaocheng People's Hospital, 67 Dongchang West Road, Liaocheng, Shandong 252000, P.R. China

E-mail: lc_uxy@126.com

Key words: paired box protein 6, microRNA-758, retinoblastoma, PI3K/Akt pathway for $\sim 2-4 \%$ of all childhood malignancies (2). An estimated 9,000 novel RB cases occur each year globally (3). Untreated RB usually rapidly progresses to blindness and is fatal because RB can spread into the brain via the optic nerve (4). To date, surgical operations, such as enucleation, combined with chemotherapy and radiotherapy are the predominant therapeutic methods for treating patients with RB $(5,6)$. Despite considerable development in diagnosis and therapy, the long-term survival of patients with RB remains unfavourable (7). Deregulations of oncogenes or tumour suppressors are implicated in RB occurrence and development; however, the pathogenesis of RB remains poorly understood $(8,9)$. Hence, identifying the molecular mechanisms by which RB occurs, determining factors that affect RB development and developing novel promising therapeutic strategies to improve the prognosis of patients with this fatal disease are urgently needed.

MicroRNAs (miRNAs), a large group of single-stranded non-coding small RNA molecules, have emerged as important gene regulators by binding to the 3'-untranslated regions (UTRs) of their target genes in a base-pairing manner, thereby promoting the formation of a miRNA-mRNA-induced silencing complex and resulting in the degradation and/or translational suppression of the target mRNAs (10). In the human genome, $>1,000$ mature miRNAs have been identified, and these miRNAs are estimated to regulate $\sim 30 \%$ of human protein-coding genes (11). miRNAs may act as tumour suppressors or oncogenes, in which aberrantly downregulated miRNAs play tumour-suppressive roles, whereas highly expressed miRNAs serve oncogenic roles in carcinogenesis and cancer progression (12). Emerging studies have indicated that numerous miRNAs are dysregulated in RB, such as miR-29a (13), miR-320 (14), miR-613 (15) and miR-143 (16). Aberrantly expressed miRNAs are closely related with RB formation and progression by regulating various pathological behaviours, such as cellular proliferation, apoptosis, invasion, metastasis and angiogenesis (17-19). Thus, in-depth investigation on miRNAs in RB may contribute to the identification of therapeutic targets for patients with this aggressive malignancy.

miR-758 has been well studied in cervical cancer (20) and hepatocellular carcinoma (21). However, the expression pattern of miR-758 in RB and its roles in the development of RB, as well as the mechanisms by which miR-758 exerts its functions, are still unknown. Therefore, this study aimed to detect miR-758 expression in RB tissues and cell lines and determine 
its effects and underlying mechanisms on RB progression. The results of this study may provide novel insights into the pathogenesis of RB and may provide an effective therapeutic target for the treatment of patients with RB.

\section{Materials and methods}

Tissue specimens. A total of $26 \mathrm{RB}$ tissues were obtained from patients (17 males and 9 females; age range, 15-53 years) with RB who received surgical resection at Liaocheng People's Hospital between September 2014 and February 2017. Normal retinal tissues were collected from 10 patients with ruptured globes. All tissues were quickly snap-frozen in liquid nitrogen and stored at $-80^{\circ} \mathrm{C}$ until RNA isolation. None of these subjects had been treated with radiotherapy or chemotherapy prior to recruitment in this study. This research was approved by the Ethics Committee of Liaocheng People's Hospital. Written informed consent was also provided by all subjects.

Cell lines. The three human RB cell lines Y79, WERI-RB1 and SO-RB50 and the normal retinal pigmented epithelial cell line ARPE-19 were acquired from the American Type Culture Collection (ATTC; Manassas, VA, USA) and cultured in Dulbecco's modified Eagle's medium (DMEM) supplemented with $10 \%$ fetal bovine serum (FBS) and $1 \%$ penicillin/streptomycin mixture (all from Gibco; Thermo Fisher Scientific, Inc., Grand Island, NY, USA). All cells were grown at $37^{\circ} \mathrm{C}$ under normoxic conditions of $95 \%$ air and $5 \% \mathrm{CO}_{2}$.

Transfection experiments. The oligonucleotides of the miR-758 mimics and their negative control (miR-NC) were chemically produced by Guangzhou RiboBio Co., Ltd. (Guangzhou, China). For PAX6 overexpression, pCMV-PAX6 plasmid and empty PAX6 plasmid were synthesised by Shanghai GenePharma Co., Ltd. (Shanghai, China). For the transfection experiments, cells were inoculated into 6-well plates at a density of $5 \times 10^{5}$ cells/well one day prior to transfection and maintained in antibiotic-free DMEM containing 10\% FBS. miRNA mimics or plasmid were transfected into cells using Lipofectamine ${ }^{\circledR} 2000$ (Invitrogen Life Technologies; Thermo Fisher Scientific, Inc., Waltham, MA, USA) following the manufacturer's instructions.

Reverse transcription-quantitative polymerase chain reaction $(R T-q P C R)$ analysis. TRIzol ${ }^{\circledR}$ reagent (Invitrogen Life Technologies; Thermo Fisher Scientific, Inc.) was applied to isolate total RNA from tissue samples or cells. The quality and concentration of total RNA was determined using a NanoDrop 2000 spectrophotometer (Thermo Fisher Scientific, Inc.). For the quantification of miR-758 expression, total RNA was converted into cDNA using a TaqMan ${ }^{\circledR}$ MicroRNA Reverse Transcription kit (Applied Biosystems; Thermo Fisher Scientific, Inc.). Subsequently, miR-758 expression was detected by performing qPCR using a TaqMan MicroRNA Assay kit on an ABI 7300 Plus thermal cycler (both from Applied Biosystems; Thermo Fisher Scientific, Inc.). U6 snRNA served as an internal control for miR-758 expression. The standard SYBR-Green RT-PCR kit (Takara Biotechnology Co., Ltd., Dalian, China) was used to quantify the mRNA level of PAX6, with $\beta$-actin employed as an internal control. Relative expression was analysed using the $2^{-\Delta \Delta \mathrm{Cq}}$ method (22).

Cell Counting Kit-8 (CCK-8) and colony formation assays. After transfection for $24 \mathrm{~h}$, cells were harvested and seeded in 96-well plates with a density of 3,000 cells/well. Following various incubation times (0-72 h), a CCK-8 assay was conducted to detect cell proliferative ability. Briefly, $10 \mu \mathrm{l}$ of CCK-8 solution (Dojindo Molecular Technologies, Inc., Kumamoto, Japan) was added into each well and further incubated at $37^{\circ} \mathrm{C}$ for $2 \mathrm{~h}$. Finally, cell proliferation was determined by detecting the absorbance at a wavelength of $450 \mathrm{~nm}$ on a multifunction microplate reader (Bio-Rad Laboratories, Inc., Hercules, CA, USA).

Transfected cells were harvested at $24 \mathrm{~h}$ post-transfection and prepared into a cell suspension. A total of 1,000 transfected cells were transferred into 6-well plates and incubated for 2 weeks at $37^{\circ} \mathrm{C}$ under $95 \%$ air and $5 \% \mathrm{CO}_{2}$. On day 15 , the cells were fixed in $4 \%$ paraformaldehyde, stained with methyl violet (Nanjing KeyGen Biotech Co., Ltd., Nanjing, China) and washed with phosphate-buffered saline (PBS). The number of colonies ( $>50$ cells/colony) was counted using an inverted microscope (Olympus IX53; Olympus Corp., Tokyo, Japan).

Detection of cell apoptosis by flow cytometric analysis. An Annexin V fluorescein isothiocyanate (FITC) Apoptosis Detection kit (BioLegend, San Diego, CA, USA) was employed to evaluate cell apoptosis. Briefly, transfected cells were collected at $48 \mathrm{~h}$ post-transfection, washed with cold PBS and suspended in $100 \mu \mathrm{l}$ of binding buffer. Subsequently, the cells were further incubated with $500 \mu \mathrm{l}$ of Annexin V-FITC and $5 \mu \mathrm{l}$ of propidium iodide (PI) in the dark at room temperature for $15 \mathrm{~min}$. Finally, cell apoptosis was detected by flow cytometry (FACScan; BD Biosciences, Franklin Lakes, NJ, USA) within $1 \mathrm{~h}$ and analysed with CellQuest software version 3.3 (BD Biosciences).

Migration and invasion assays. Transwell chambers with 8- $\mu \mathrm{m}$ pore size (Corning Inc., Corning, NY, USA) coated with or without Matrigel (BD Biosciences) were used for the detection of cellular migration and invasion, respectively.

At $48 \mathrm{~h}$ post-transfection, a total of $1 \times 10^{5}$ transfected cells were suspended in FBS-free DMEM and placed into the top chambers. The bottom chambers were filled with $5 \mu \mathrm{l}$ of DMEM containing $10 \% \mathrm{FBS}$. After $24 \mathrm{~h}$ of incubation at $37^{\circ} \mathrm{C}$ with $95 \%$ air and $5 \% \mathrm{CO}_{2}$, non-migrated and non-invaded cells were removed from the surface of the membrane with a cotton swab. The migrated and invaded cells were fixed with $100 \%$ methanol, stained with $0.5 \%$ crystal violet, washed with PBS and photographed using an inverted microscope. Values for migration and invasion were determined by counting five randomly selected fields per membrane.

Bioinformatics analysis. TargetScan online software (www.targetscan.org) and microRNA.org (www.microrna. org $/$ microrna/) were used to predict the potential targets of miR-758.

Luciferase reporter assay. PAX6 was predicted as a major target gene of miR-758, and this association was further 

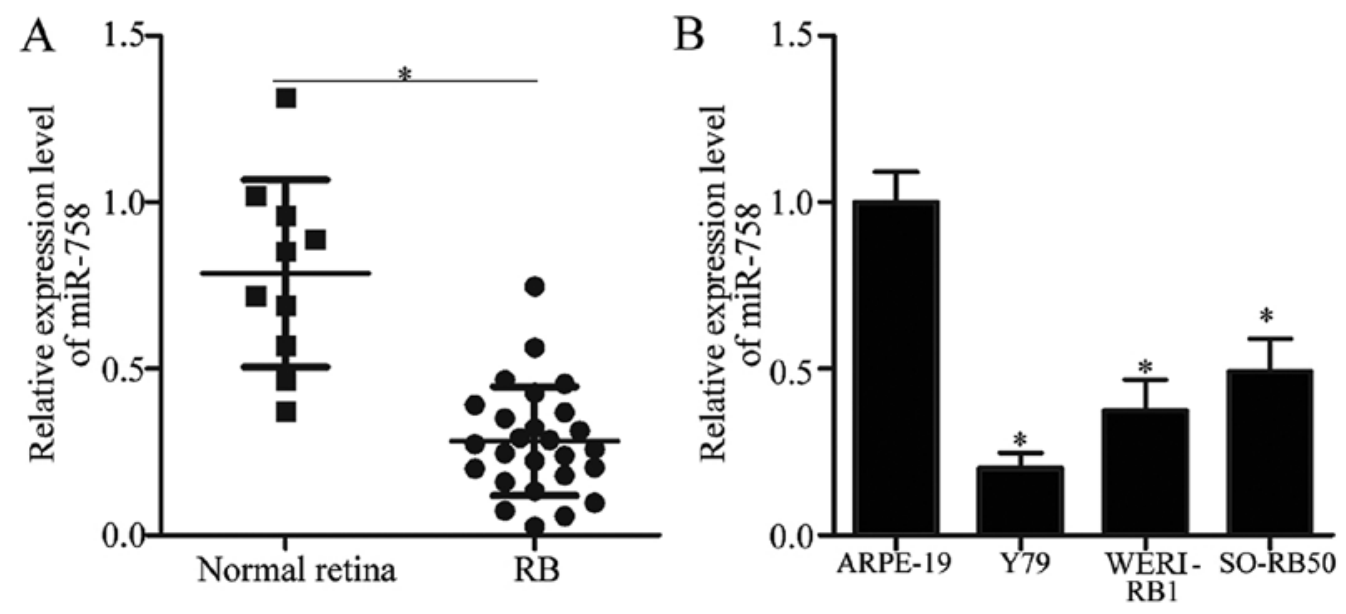

Figure 1. The expression of miR-758 is reduced in RB tissues and cell lines. (A) The expression level of miR-758 in 26 RB tissues and 10 normal retinal tissues was determined by RT-qPCR. "P $<0.05$ vs. the normal retinal tissues. (B) The expression of miR-758 in three RB cell lines and one normal retinal pigmented epithelial cell line was assessed by RT-qPCR. " $\mathrm{P}<0.05$ vs. ARPE-19.

confirmed by luciferase reporter assay. To synthesise luciferase reporter plasmids, we amplified the 3'-UTR fragment of PAX6 containing wild-type $(\mathrm{Wt})$ putative or mutant-type binding sites for miR-758 (GenePharma Co., Ltd.), inserted it into the pmirGLO luciferase reporter vector (Promega Corp., Madison, WI, USA) and labelled it as pmirGLO-Wt-PAX6-3'-UTR or pmirGLO-Mut-PAX6-3'-UTR, respectively. For the luciferase assay, the cells were seeded into 24 -well plates and co-transfected with miR-758 mimics or miR-NC and pmirGLO-Wt-PAX6-3'-UTR or pmirGLO-Mut-PAX6-3'-UTR using Lipofectamine ${ }^{\circledR} 2000$, in accordance with the manufacturer's protocol. Luciferase activities were detected at $48 \mathrm{~h}$ after transfection using a Dual-Luciferase Reporter Assay System (Promega Corp.). Renilla luciferase activity was determined for normalisation.

Western blot analysis. The total protein of cells or tissues was extracted using ice-cold radioimmunoprecipitation assay buffer (Beyotime Institute of Biotechnology, Shanghai, China). The concentration of total protein was quantified with a BCA Protein Assay kit (Beyotime Institute of Biotechnology). Equal amounts of protein were separated by $10 \%$ SDS-PAGE and then transferred to polyvinylidene difluoride membranes (PVDF; EMD Millipore, Billerica, MA, USA). Subsequently, the membranes were blocked with 5\% skimmed dry milk in Tris-buffered saline-Tween (TBST) at room temperature for $2 \mathrm{~h}$ and incubated with primary antibodies at $4^{\circ} \mathrm{C}$ overnight. After washing with TBST thrice, the membranes were incubated with goat anti-mouse horseradish peroxidase-conjugated secondary antibodies (1:5,000 dilution; cat. no. sc-2005; Santa Cruz Biotechnology, Inc., Santa Cruz, CA, USA) or goat anti-rabbit horseradish peroxidase-conjugated secondary antibodies (1:5,000 dilution; cat. no. sc-2004; Santa Cruz Biotechnology, Inc.) at room temperature for $1 \mathrm{~h}$. The membranes were washed thrice with TBST and subjected to protein signal detection using an enhanced chemiluminescence reagent (Bio-Rad Laboratories). The primary antibodies used in the present study included mouse anti-human monoclonal PAX6 (1:500 dilution; cat. no. ab197768), rabbit anti-human monoclonal p-pi3k (1:500 dilution; cat. no. ab182651) and mouse anti-human monoclonal pi3k (1:500 dilution; cat. no. ab86714; all from Abcam, Cambridge, UK), mouse anti-human monoclonal p-Akt (1:1,000 dilution; cat. no. sc-81433) and mouse anti-human monoclonal Akt (1:1,000 dilution; cat. no. sc-56878; both from Santa Cruz Biotechnology, Inc.), and rabbit anti-human monoclonal GAPDH antibody (1:500 dilution; cat. no. ab181603; Abcam). GAPDH was employed as the internal reference.

Statistical analysis. The data were expressed as the median \pm standard deviation (SD) and analysed with SPSS 17.0 (SPSS, Inc., Chicago, IL, USA). Differences between groups were compared with Student's t-test or one-way ANOVA, followed by the SNK multiple comparison test. The possible correlation between miR-758 and PAX6 mRNA in RB tissues was evaluated using Spearman's correlation analysis. $\mathrm{P}<0.05$ indicated a statistically significant difference.

\section{Results}

miR-758 is downregulated in RB tissues and cell lines. To illustrate the potential roles of miR-758 in RB, we first detected miR-758 expression in $26 \mathrm{RB}$ tissues and 10 normal retinal tissues. The RT-qPCR analysis data revealed that miR-758 was significantly downregulated in RB tissues compared with that in normal retinal tissues (Fig. $1 \mathrm{~A}, \mathrm{P}<0.05$ ). In addition, miR-758 expression level was determined in the three RB cell lines (Y79, WERI-RB1 and SO-RB50) and in the normal retinal pigmented epithelium cell line ARPE-19. Compared with that in ARPE-19, the expression levels of miR-758 were markedly reduced in all three RB cell lines (Fig. 1B, $\mathrm{P}<0.05$ ). These results indicated that the dysregulation of miR-758 may be involved in the progression of RB.

MiR-758 overexpression inhibits proliferation and promotes apoptosis of RB cells. To explore the role of miR-758 in the progression of $\mathrm{RB}$, we transfected miR-758 mimics into Y79 and WERI-RB1 cells to upregulate the endogenous miR-758 expression level. Following transfection, miR-758 was markedly overexpressed in Y79 and WERI-RB1 cells 

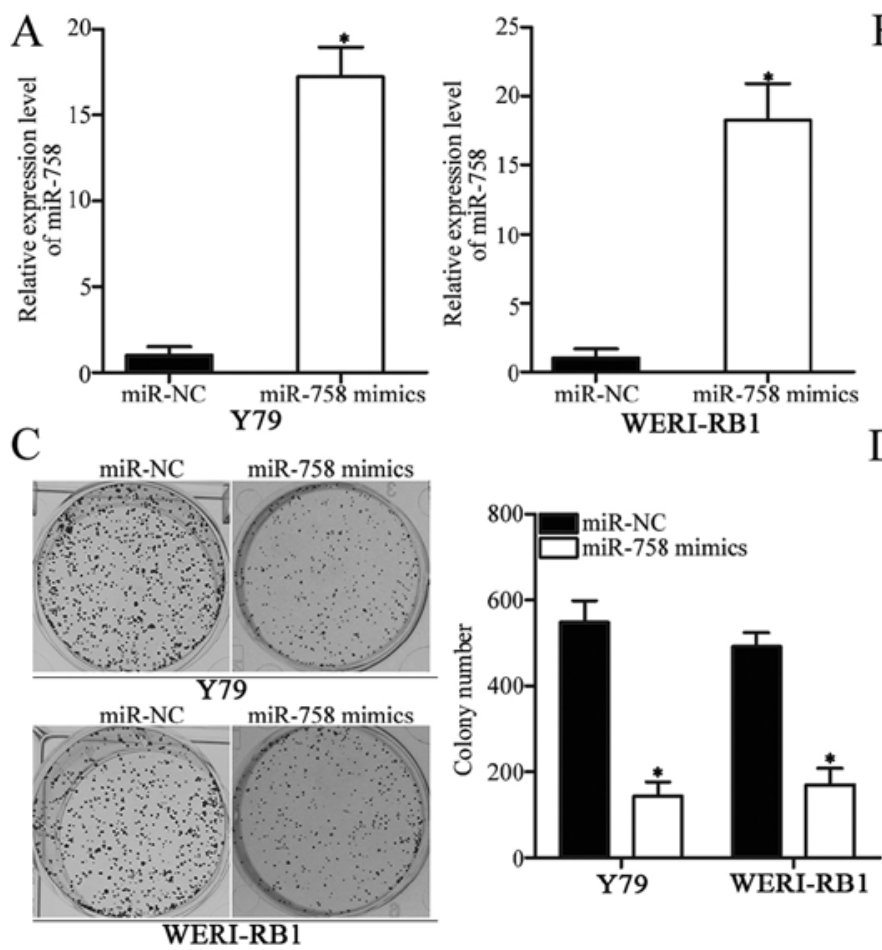
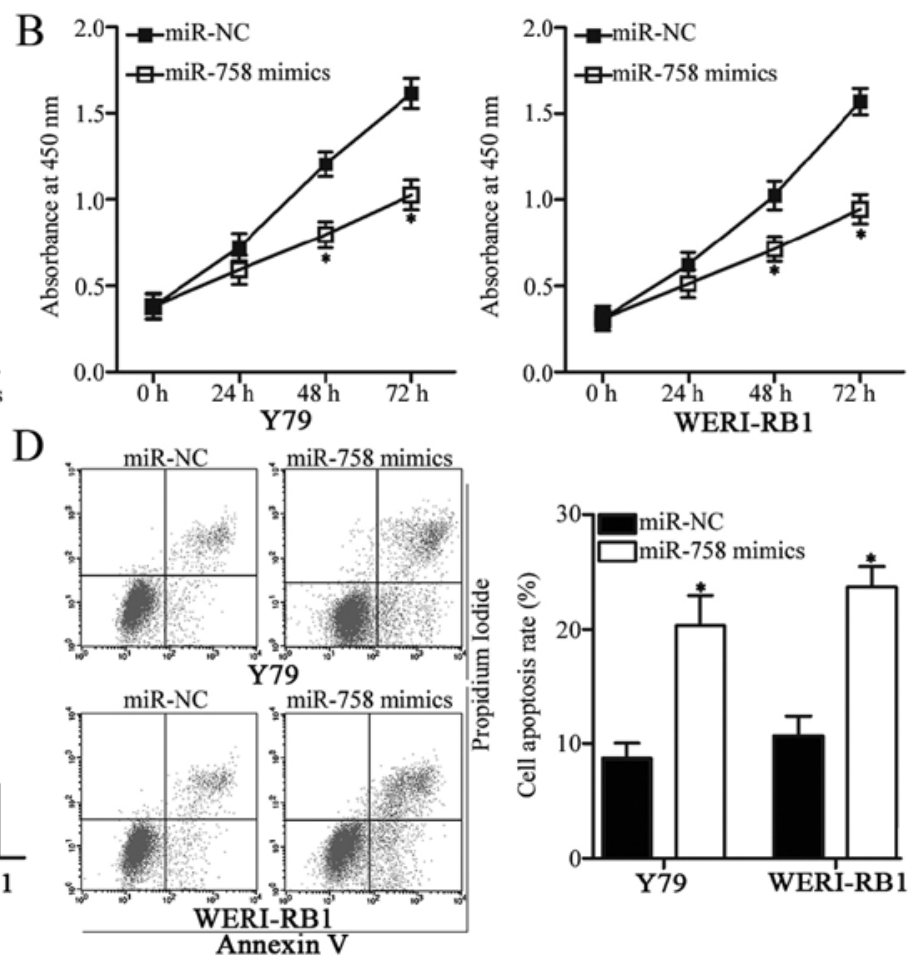

Figure 2. Ectopic expression of miR-758 attenuates proliferation and induces apoptosis of Y79 and WERI-RB1 cells. (A) RT-qPCR was performed to detect miR-758 expression after the transfection of Y79 and WERI-RB1 cells with miR-758 mimics or miR-NC. "P<0.05 vs. miR-NC. (B and C) CCK-8 and colony formation assays were employed to determine the effects of miR-758 overexpression on $\mathrm{Y} 79$ and WERI-RB1 cell proliferation. ${ }^{*} \mathrm{P}<0.05$ vs. miR-NC. (D) The apoptosis rate in Y79 and WERI-RB1 cells transfected with miR-758 mimics or miR-NC was evaluated using a flow cytometric assay. ${ }^{*} \mathrm{P}<0.05$ vs. miR-NC.

transfected with miR-758 mimics compared with that in cells transfected with miR-NC (Fig. 2A, P<0.05). The effect of miR-758 overexpression on cellular proliferation was investigated using a CCK-8 assay. As displayed in Fig. 2B, the proliferation of $\mathrm{Y} 79$ and WERI-RB1 cells transfected with miR-758 mimics was delayed compared with that in cells transfected with miR-NC $(\mathrm{P}<0.05)$.

Colony formation assay was performed to further confirm the inhibition of RB cell proliferation caused by miR-758 overexpression. The results revealed that the colony numbers of Y79 and WERI-RB1 cells transfected with miR-758 mimics were significantly decreased relative to those in the miR-NC groups (Fig. 2C, $\mathrm{P}<0.05$ ). Furthermore, flow cytometric assay revealed that the enforced expression of miR-758 increased the percentage of cell apoptosis in Y79 and WERI-RB1 cells (Fig. 2D, $\mathrm{P}<0.05$ ). These findings revealed that miR-758 may be important in regulating the proliferation and apoptosis of RB cells.

Overexpression of the expression of miR-758 inhibits cell migration and invasion in $R B$ cells. Migration and invasion assays were employed to determine the role of miR-758 on cell metastasis in RB cells. As shown in Fig. 3A, overexpression of miR-758 suppressed the migratory capacities of $\mathrm{Y} 79$ and WERI-RB1 cells compared with those in the miR-NC groups $(\mathrm{P}<0.05)$. Similarly, the number of invaded cells was lower in the miR-758 mimics-transfected Y79 and WERI-RB1 cells than those in cells transfected with miR-NC (Fig. 3B, $\mathrm{P}<0.05)$. These results indicated that miR-758 may have suppressive roles in RB metastasis.
PAX6 is direct target of miR-758 in RB. To gain insights into the mechanisms underlying the action of miR-758 in RB, we conducted bioinformatics analysis to predict the potential targets of miR-758. PAX6, a major highly conserved putative target of miR-758, plays oncogenic roles in RB (23-25) and was therefore selected for further confirmation (Fig. 4A). To clarify whether miR-758 can directly interact with the 3'-UTR of PAX6, we performed luciferase reporter assays in Y79 and WERI-RB1 cells transfected with miR-758 mimics or miR-NC in the presence of pmirGLO-Wt-PAX6-3'-UTR or pmirGLO-Mut-PAX6-3'-UTR. Luciferase activities were considerably reduced in Y79 and WERI-RB1 cells co-transfected with miR-758 mimics and the luciferase reporter plasmid carrying the wild-type miR-758 binding sequences $(\mathrm{P}<0.05)$. However, miR-758 upregulation did not affect the luciferase activities of the plasmid carrying the mutant PAX6 3'-UTR (Fig. 4B). To further determine the regulatory roles of miR-758 on endogenous PAX6 expression, we utilised RT-qPCR and western blot analysis to assess the expression levels of PAX6 in Y79 and WERI-RB1 cells after transfection with miR-758 mimics or miR-NC. The ectopic expression of miR-758 decreased the mRNA (Fig. 4C, P<0.05) and protein (Fig. 4D, P<0.05) expression of PAX6 in both Y79 and WERI-RB1 cells. In summary, these data demonstrated that PAX6 is a direct target of miR-758 in RB.

Inverse correlation between PAX6 and miR-758 expression in $R B$ tissues. To examine the possible clinical relevance of PAX6 in $\mathrm{RB}$, we detected PAX6 mRNA and protein expression in RB and normal retinal tissues. As indicated in Fig. 5A and B, the 
A

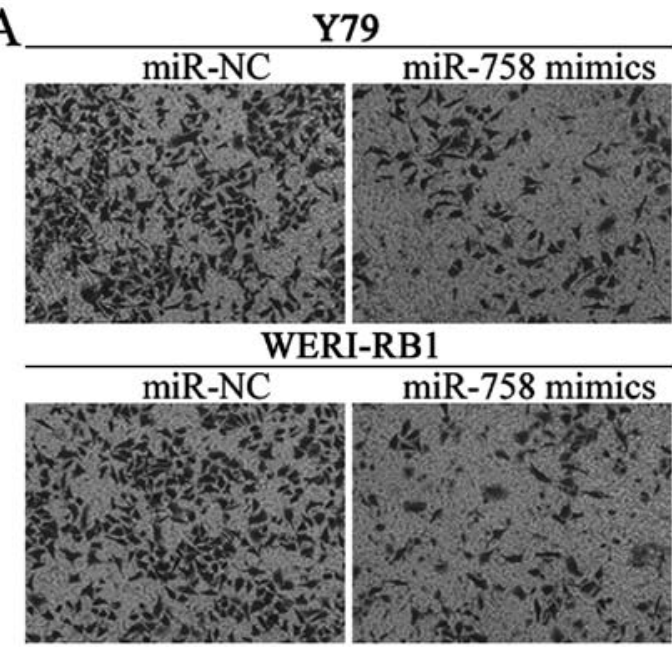

B

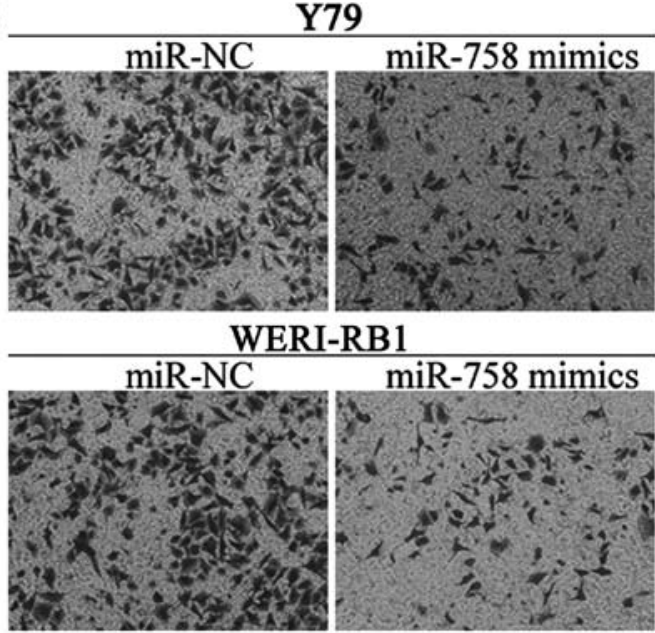

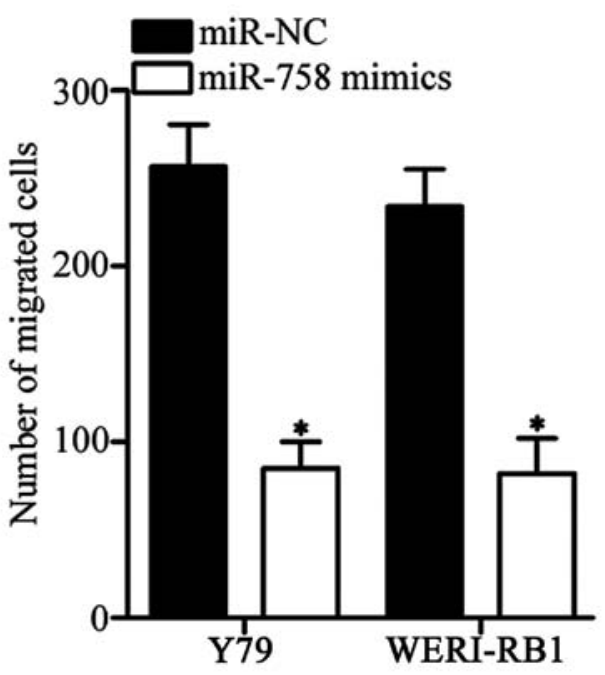

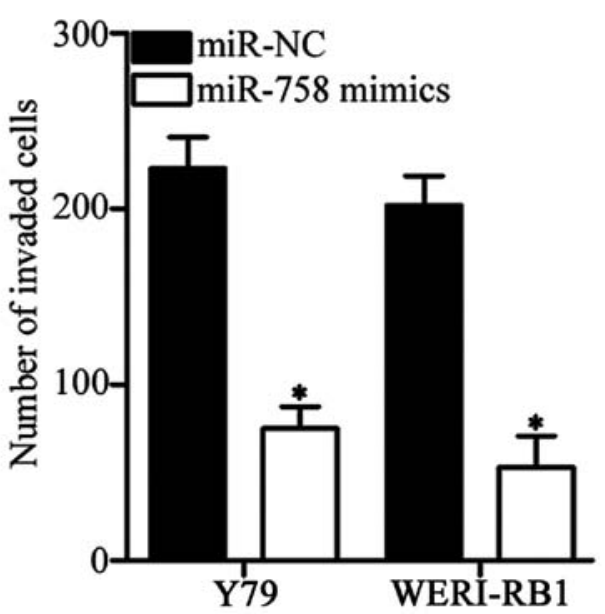

Figure 3. Upregulation of miR-758 decreases the migration and invasion of Y79 and WERI-RB1 cells. Y79 and WERI-RB1 cells were transfected with miR-758 mimics or miR-NC. Following $48 \mathrm{~h}$ of transfection, (A) cell migration and (B) invasive abilities were determined by Transwell migration and invasion assays. ${ }^{*} \mathrm{P}<0.05$ vs. miR-NC.

mRNA and protein levels of PAX6 were significantly higher in the $\mathrm{RB}$ tissues than those in the normal retinal tissues $(\mathrm{P}<0.05)$. We analysed the correlation between miR-758 and PAX6 mRNA in RB tissues using Spearman's correlation analysis. An inverse correlation was validated between miR-758 and PAX6 mRNA level in RB tissues (Fig. 5C; $r=-0.5946$, $\mathrm{P}=0.0014)$. These data further indicated that PAX6 is a direct target of miR-758 in RB.

PAX6 overexpression abrogates the impact of miR-758 overexpression on $R B$ cells. After determining that PAX6 is a direct target of miR-758, we further performed a series of rescue experiments to investigate whether miR-758 mediates its tumour-suppressive roles in RB cells by inhibiting PAX6. PAX6 overexpression plasmid pCMV-PAX6 or empty pCMV plasmid, together with miR-758 mimics, were transfected into Y79 and WERI-RB1 cells. After transfection, western blot analysis confirmed that the decreased PAX6 protein induced by miR-758 overexpression was restored in Y79 and WERI-RB1 cells after co-transfection with pCMV-PAX6 (Fig. 6A, $\mathrm{P}<0.05)$. Functional assays revealed that restored PAX6 expression significantly abrogated the effects of miR-758 overexpression on cell proliferation (Fig. 6B, $\mathrm{P}<0.05$ ), colony formation (Fig. 6C, P<0.05), apoptosis (Fig. 6D, P<0.05), migration (Fig. 6E, $\mathrm{P}<0.05$ ) and invasion (Fig. 6F, $\mathrm{P}<0.05$ ). These results revealed that miR-758 may exert tumour-suppressive effects in RB cells, at least partly, by downregulating PAX6 expression.

miR-758 inactivates the PI3K/Akt pathway in RB cells. PAX6 participates in the regulation of the PI3K/Akt pathway $(26,27)$. Therefore, we investigated whether miR-758 could regulate the PI3K/Akt pathway in RB cells via PAX6 modulation. Y79 and WERI-RB1 cells were transfected with miR-758 mimics in the presence of pCMV-PAX6 or pCMV. Following transfection for $72 \mathrm{~h}$, the ectopic expression of miR-758 significantly reduced $\mathrm{p}-\mathrm{PI} 3 \mathrm{~K}$ and $\mathrm{p}$-Akt protein levels in Y79 and WERI-RB1 cells relative to those in the miR-NC groups. Furthermore, the downregulation of p-PI3K and p-Akt protein levels caused by miR-758 overexpression was restored in Y79 and WERI-RB1 cells after co-transfection with pCMV-PAX6 (Fig. 7). These results confirmed that miR-758 may act as a tumour suppressor in $\mathrm{RB}$ by negatively regulating the PAX6/PI3K/Akt pathway. 


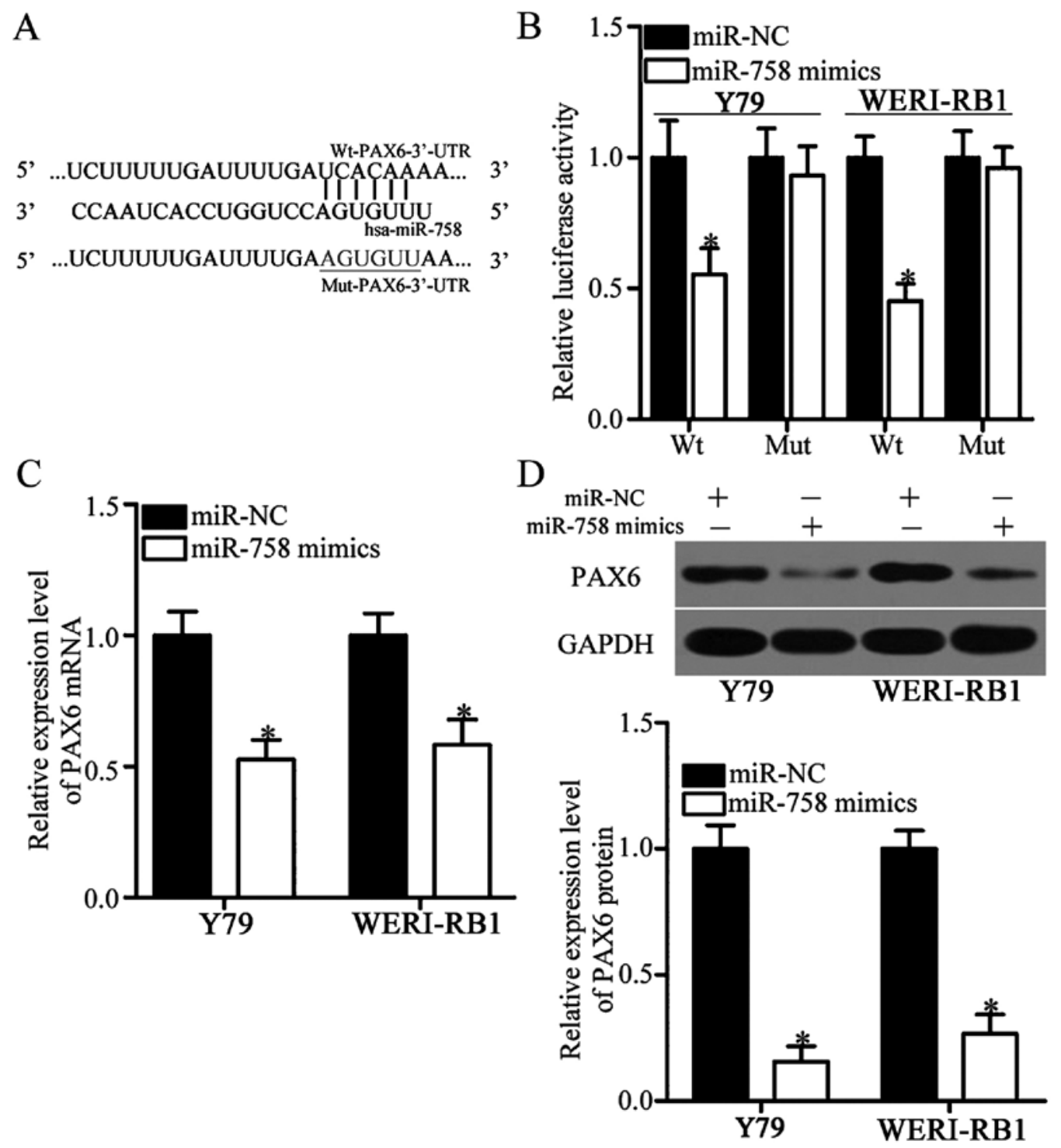

Figure 4. PAX6 is a direct target gene of miR-758. (A) Predicted wild-type (Wt) and mutant (Mut) miR-758 binding sites in the 3'-UTR of PAX6. (B) Y79 and WERI-RB1 cells were co-transfected with miR-758 mimics or miR-NC and a luciferase reporter plasmid containing Wt or Mut 3'-UTR of PAX6. Following $48 \mathrm{~h}$ of transfection, firefly and Renilla luciferase activities were detected using a Dual-Luciferase Reporter Assay system. "P<0.05 vs. miR-NC. (C and D) RT-qPCR and western blot analysis of PAX6 mRNA and protein expression in Y79 and WERI-RB1 cells transfected with miR-758 mimics or miR-NC. ${ }^{*} \mathrm{P}<0.05$ vs. miR-NC.
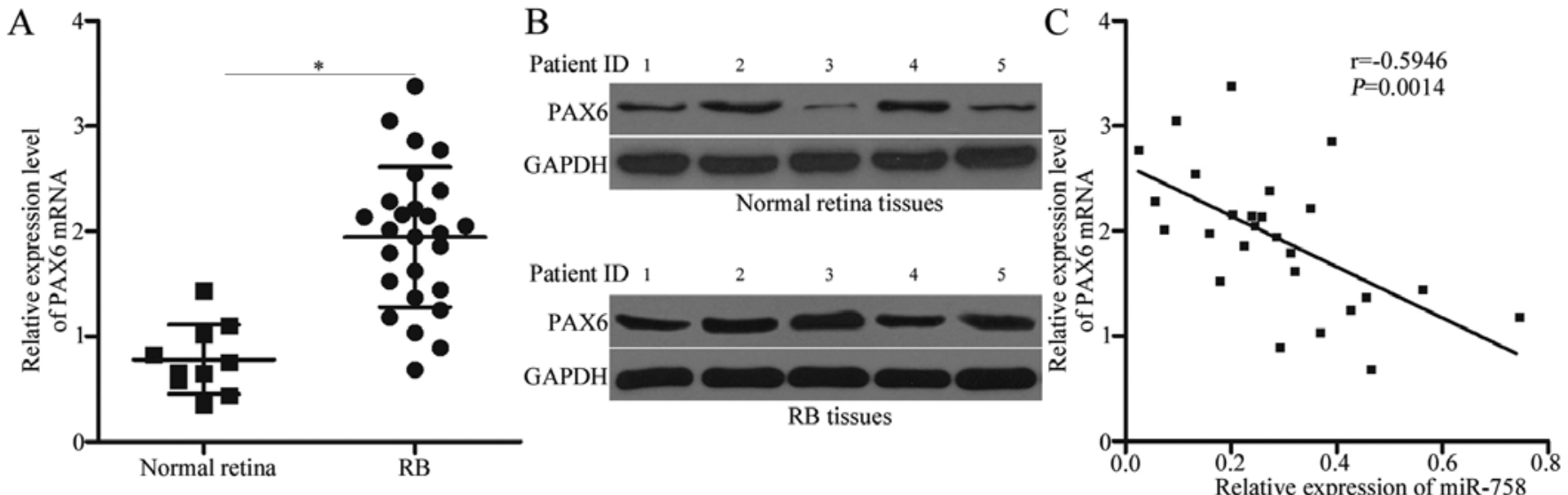

Figure 5. PAX6 is overexpressed and inversely correlated with miR-758 expression in RB tissues. (A) PAX6 mRNA expression was detected by RT-qPCR in $26 \mathrm{RB}$ tissues and 10 normal retinal tissues. " $\mathrm{P}<0.05$ vs. the normal retinal tissues. (B) PAX6 protein expression was determined in several RB tissues and normal retinal tissues using western blot analysis. (C) Spearman's correlation analysis was used to assess the correlation between miR-758 and PAX6 mRNA expression level in RB tissues. $\mathrm{r}=-0.5946, \mathrm{P}=0.0014$. 
A

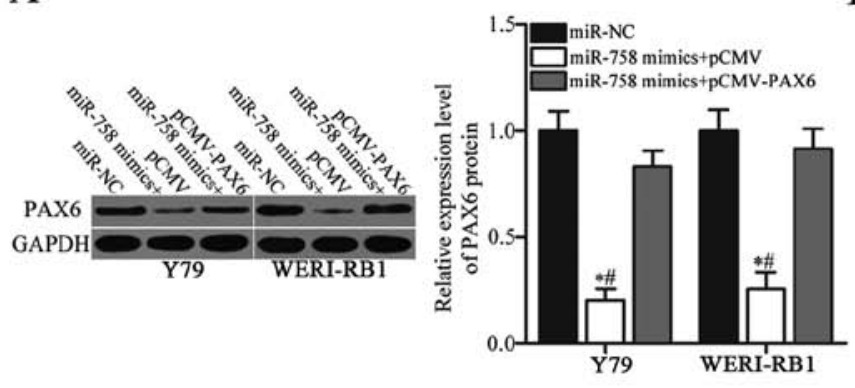

B

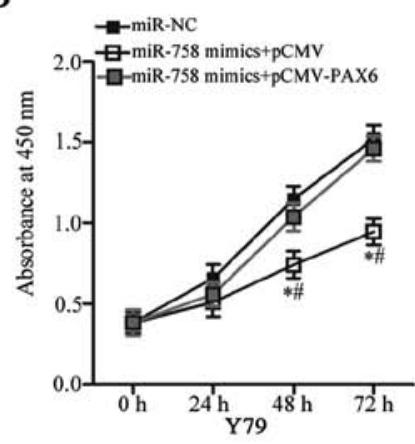

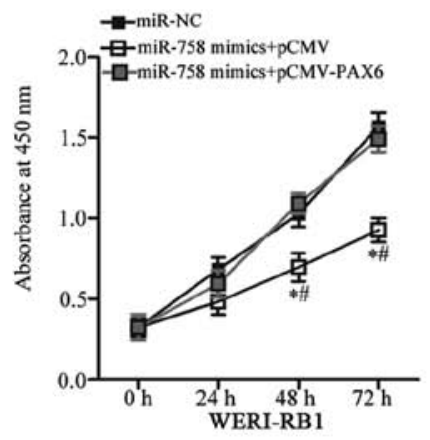

C
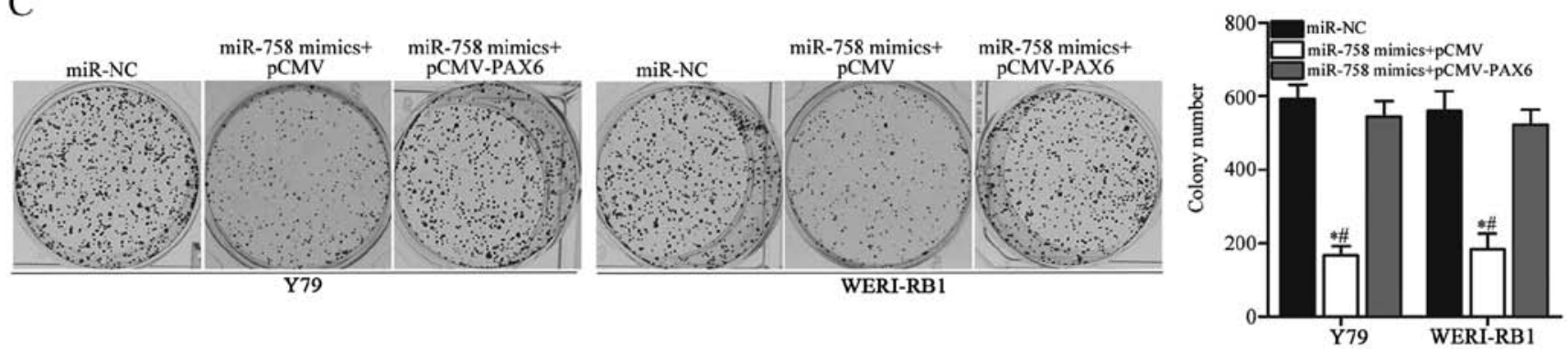

D
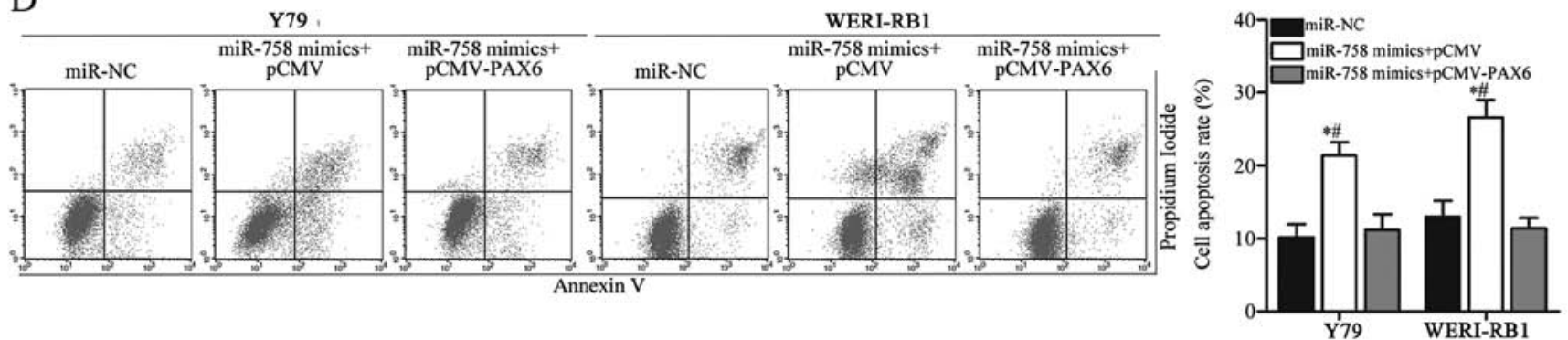

E
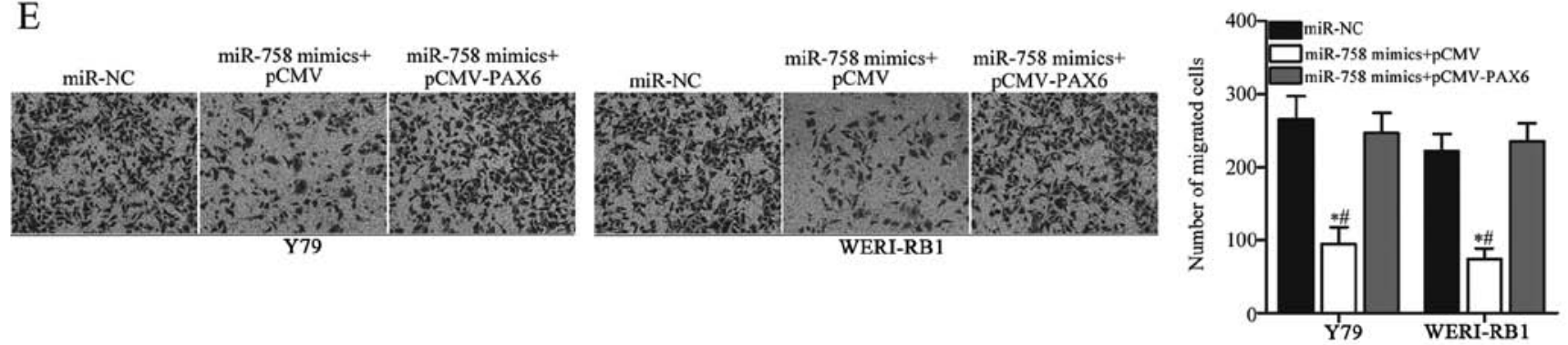

F
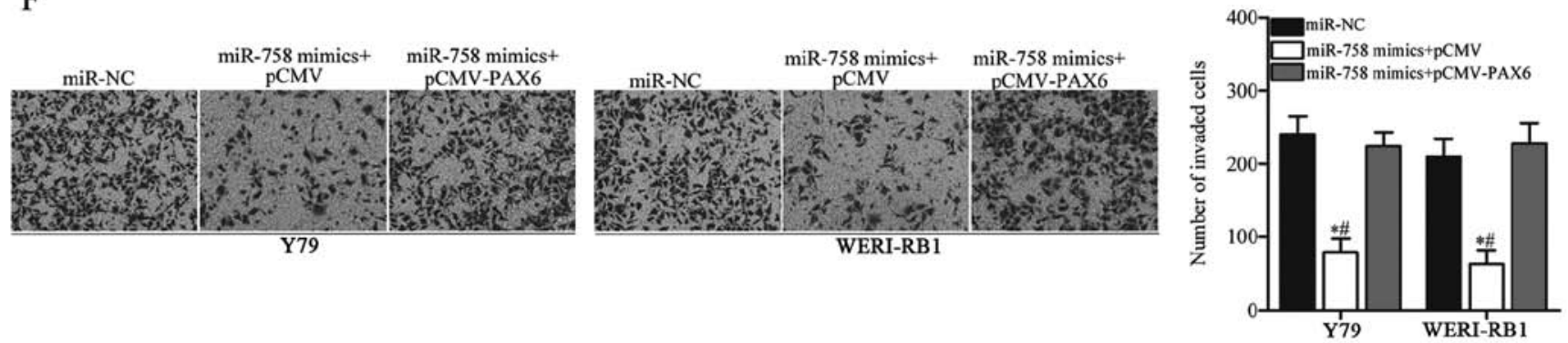

Figure 6. PAX6 mediates multiple biological actions of miR-758 overexpression in RB cells. Y79 and WERI-RB1 cells were co-transfected with miR-758 mimics and pCMV-PAX6 or pCMV and were used in the following experiments. (A) After $72 \mathrm{~h}$ of transfection, PAX6 protein levels in indicated cells were detected by western blot analysis. ${ }^{*} \mathrm{P}<0.05$ vs. miR-NC. ${ }^{*} \mathrm{P}<0.05$ vs. miR-758 mimics+pCMV-PAX6. (B-D) Cell proliferation, colony formation and apoptosis in the aforementioned cells were evaluated using CCK- 8 , colony formation and flow cytometric assays, respectively. ${ }^{*} \mathrm{P}<0.05$ vs. miR-NC. ${ }^{*} \mathrm{P}<0.05$ vs. miR-758 mimics+pCMV-PAX6. (E and F) Migration and invasion assays were applied to determine cell migration and invasion in the aforementioned cells, respectively. "P<0.05 vs. miR-NC. "P<0.05 vs. miR-758 mimics+pCMV-PAX6. 

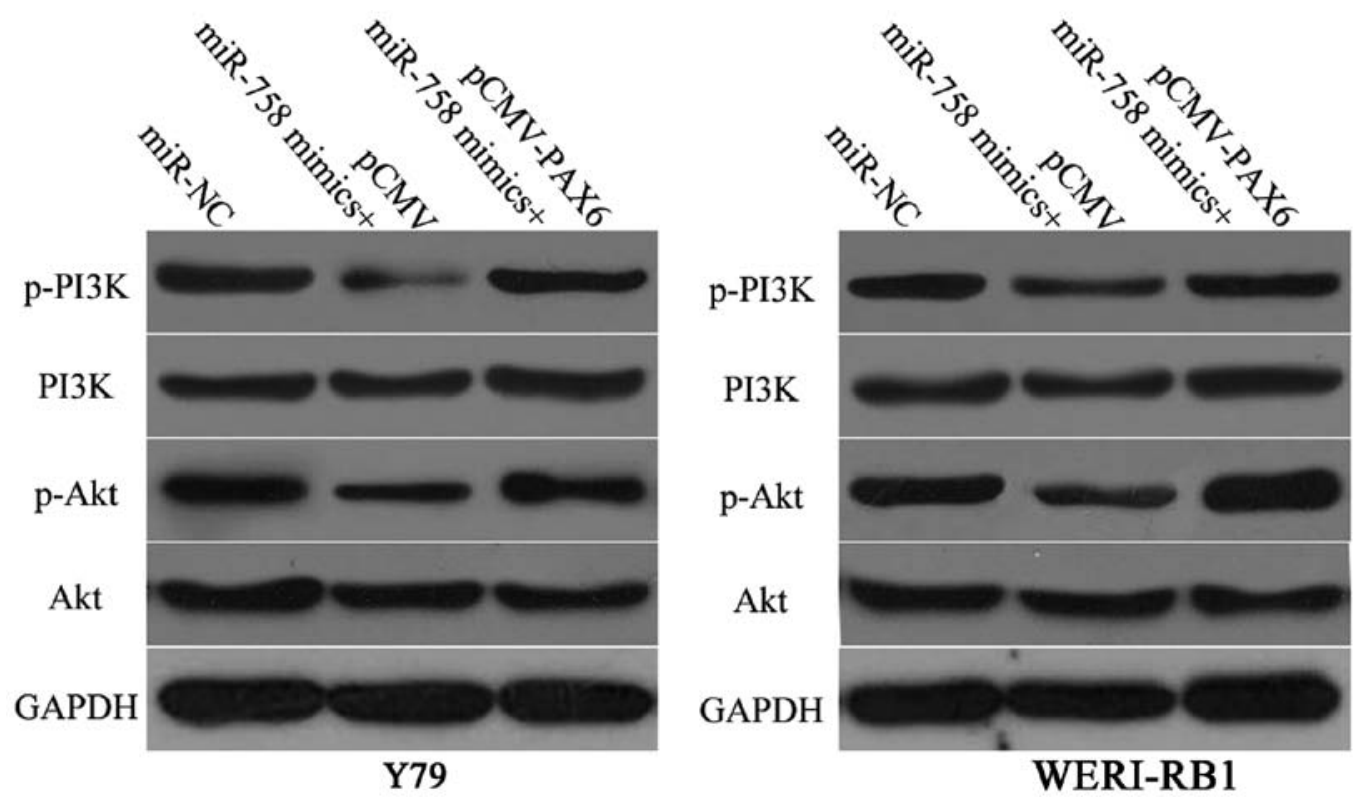

Figure 7. Ectopic miR-758 expression inhibits PI3K/Akt pathway activation by regulating PAX6 expression in RB cells. miR-758 mimics, along with pCMV-PAX6 or pCMV, were introduced in Y79 and WERI-RB1 cells. Western blot analysis was conducted at $72 \mathrm{~h}$ post-transfection to quantify p-PI3K, PI3K, p-Akt and Akt protein levels.

\section{Discussion}

In recent decades, accumulated studies have highlighted that the dysregulation of miRNAs in RB is a leading cause for tumourigenesis and tumour development (28-30). Therefore, the elucidation of the expression, functional roles and underlying mechanisms of miRNAs in RB would help develop promising therapeutic methods to improve the prognosis of RB patients. miR-758 is downregulated in cervical cancer, and this downregulation is correlated with infiltration and invasion of cervical cancer (20). Jiang et al (21) reported that miR-758 was downregulated in hepatocellular carcinoma tissues and cell lines. Enforced expression of miR-758 decreased cell growth and metastasis in hepatocellular carcinoma. However, the expression pattern and detailed roles of miR-758 in RB remain unknown. Thus, the present study aimed to detect miR-758 expression in RB and determine the biological roles and underlying mechanisms of miR-758 in RB cells and tissues.

The key finding in this study is that miR-758 was downregulated in RB tissues and cell lines compared with that in normal retinal tissues and a normal retinal pigmented epithelium cell line, respectively. Overexpression of miR-758 inhibited cellular proliferation, migration and invasion and promoted apoptosis in RB cells in vitro. In addition, PAX6 was confirmed as a direct target of miR-758 in RB. PAX6 was overexpressed in RB tissues and negatively correlated with the expression level of miR-758. Overexpression of PAX6 rescued the tumor-suppressive roles of miR-758 overexpression in RB cells. Notably, ectopic expression of miR-758 inhibited the activation of the PI3K/Akt pathway in RB cells by inhibiting PAX6. To the best of our knowledge, our present study is the first to demonstrate that miR-758 exerts tumour-suppressive effects on the malignant phenotypes of RB cells, at least in part, by directly targeting PAX6 and inactivating the PI3K/Akt pathway. These findings also suggest that the miR-758/PAX6 axis may be developed as a potential therapeutic target for patients with this aggressive disease.

Recognising the targets of miR-758 in RB is essential to understand the mechanisms on the regulatory roles of miR-758 in RB occurrence and development. It is also important for the identification of novel therapeutic targets for patients with RB. Several targets of miR-758 have been identified, including MDM2 (21), mTOR (21), CD36 (31) and ABCA1 (32). In the present study, PAX6 was demonstrated to be a novel target of miR-758 in RB. PAX6, a highly conserved transcription factor, is a member of the PAX gene family (33). It plays important roles in the development of the eyes, central nervous system and pancreas $(34,35)$. Emerging studies have demonstrated that PAX6 is implicated in the carcinogenesis and progression of multiple human cancers. For example, PAX6 is overexpressed in breast cancer tissues and cell lines. Breast cancer patients with an increased expression level of PAX6 exhibited poorer prognosis than those with a decreased PAX6 level (36). PAX6 knockdown was revealed to inhibit cell viability, proliferation, colony formation and metastasis in vitro and decrease tumour growth in vivo $(37,38)$. Hence, PAX6 may be a promising biomarker for diagnosis and prognosis and an effective therapeutic target in tumour patients.

PAX6 is closely related with RB onset and development. It is highly expressed in RB tissues and cell lines. Its suppression was revealed to significantly reduce cell proliferation, migration, invasion, cell cycle arrest and cell apoptosis in RB cells (23-25). PAX6 is directly targeted by several miRNAs in human cancers. For example, miR-433 (25) and miR-365b-3p (39) directly target PAX6 to inhibit the malignant progression of RB. Furthermore, miR-223 (27), miR-7 (40), miR-335 (33) and miR-448 (41) directly bind to PAX6 and therefore inhibit the progression of glioblastoma, non-small cell lung, breast and gastric cancer, respectively. 
These findings indicated that miRNA-based therapy targeting PAX6 may represent a novel therapeutic method for the treatment of tumour patients.

In conclusion, the present study provides new evidence that miR-758 may play tumour-suppressive roles in $\mathrm{RB}$ by directly targeting PAX6 and regulating the PI3K/Akt pathway. Our findings regarding miR-758 are encouraging and suggest that this miRNA can be explored as a potential therapeutic target for patients with $\mathrm{RB}$ in the future. However, we did not analyze the correlation between miR-758 expression and the prognosis of patients with RB. This is a limitation of the present study and we will explore this in our future experiments.

\section{Acknowledgements}

Not applicable.

\section{Funding}

No funding was received.

\section{Availability of data and materials}

The datasets used and/or analyzed during the present study are available from the corresponding author on reasonable request.

\section{Authors' contributions}

JL and XY designed this research, and performed all functional experiments. Both authors read and approved the manuscript and agree to be accountable for all aspects of the research in ensuring that the accuracy or integrity of any part of the work are appropriately investigated and resolved.

\section{Ethics approval and consent to participate}

This research was approved by the Ethics Committee of the Liaocheng People's Hospital. Written informed consent was also provided by all subjects.

\section{Patient consent for publication}

Not applicable.

\section{Competing interests}

The authors declare that they have no competing interests.

\section{References}

1. Dimaras H, Kimani K, Dimba EA, Gronsdahl P, White A, Chan HS and Gallie BL: Retinoblastoma. Lancet 379: 1436-1446, 2012.

2. Broaddus E, Topham A and Singh AD: Incidence of retinoblastoma in the USA: 1975-2004. Br J Ophthalmol 93: 21-23, 2009.

3. Kivelä T: The epidemiological challenge of the most frequent eye cancer: Retinoblastoma, an issue of birth and death. Br J Ophthalmol 93: 1129-1131, 2009.

4. Dong C, Liu S, Lv Y, Zhang C, Gao H, Tan L and Wang H: Long non-coding RNA HOTAIR regulates proliferation and invasion via activating Notch signalling pathway in retinoblastoma. J Biosci 41: 677-687, 2016.
5. Shields CL, Say EA, Pointdujour-Lim R, Cao C, Jabbour PM and Shields JA: Rescue intra-arterial chemotherapy following retinoblastoma recurrence after initial intra-arterial chemotherapy. J Fr Ophtalmol 38: 542-549, 2015.

6. Chantada GL, Qaddoumi I, Canturk S, Khetan V, Ma Z, Kimani K, Yeniad B, Sultan I, Sitorus RS, Tacyildiz N and Abramson DH: Strategies to manage retinoblastoma in developing countries. Pediatr Blood Cancer 56: 341-348, 2011.

7. Abramson DH, Shields CL, Munier FL and Chantada GL: Treatment of retinoblastoma in 2015: Agreement and disagreement. JAMA Ophthalmol 133: 1341-1347, 2015.

8. Beta M, Venkatesan N, Vasudevan M, Vetrivel U, Khetan V and Krishnakumar S: Identification and insilico analysis of retinoblastoma serum microrna profile and gene targets towards prediction of novel serum biomarkers. Bioinform Biol Insights 7: 21-34, 2013

9. Li J, Zhang Y, Wang X and Zhao R: microRNA-497 overexpression decreases proliferation, migration and invasion of human retinoblastoma cells via targeting vascular endothelial growth factor A. Oncol Lett 13: 5021-5027, 2017.

10. Bartel DP: MicroRNAs: Genomics, biogenesis, mechanism, and function. Cell 116: 281-297, 2004.

11. Lewis BP, Burge CB and Bartel DP: Conserved seed pairing, often flanked by adenosines, indicates that thousands of human genes are microRNA targets. Cell 120: 15-20, 2005.

12. Shenouda SK and Alahari SK: MicroRNA function in cancer: Oncogene or a tumor suppressor? Cancer Metastasis Rev 28: 369-378, 2009.

13. Liu S, Zhang X, Hu C, Wang Y and Xu C: miR-29a inhibits human retinoblastoma progression by targeting STAT3. Oncol Rep 39: 739-746, 2018.

14. Liang Y, Chen X and Liang Z: MicroRNA-320 regulates autophagy in retinoblastoma by targeting hypoxia inducible factor-1 $\alpha$. Exp Ther Med 14: 2367-2372, 2017

15. Zhang Y, Zhu X, Zhu X, Wu Y, Liu Y, Yao B and Huang Z: MiR-613 suppresses retinoblastoma cell proliferation, invasion, and tumor formation by targeting E2F5. Tumour Biol 39: 1010428317691674, 2017.

16. Wang LL, Hu HF and Feng YQ: Suppressive effect of microRNA-143 in retinoblastoma. Int J Ophthalmol 9: 1584-1590, 2016.

17. Xu X, Ge S, Jia R, Zhou Y, Song $X$, Zhang H and Fan $X$ : Hypoxia-induced miR-181b enhances angiogenesis of retinoblastoma cells by targeting PDCD10 and GATA6. Oncol Rep 33: 2789-2796, 2015

18. Liu S, Hu C, Wang Y, Shi G, Li Y and Wu H: miR-124 inhibits proliferation and invasion of human retinoblastoma cells by targeting STAT3. Oncol Rep 36: 2398-2404, 2016.

19. Yang L, Wei N, Wang L, Wang X and Liu QH: miR-498 promotes cell proliferation and inhibits cell apoptosis in retinoblastoma by directly targeting CCPG1. Childs Nerv Syst 34: 417-422, 2018.

20. Meng X, Zhao Y, Wang J, Gao Z, Geng Q and Liu X: Regulatory roles of miRNA-758 and matrix extracellular phosphoglycoprotein in cervical cancer. Exp Ther Med 14: 2789-2794, 2017.

21. Jiang D, Cho W, Li Z, Xu X, Qu Y, Jiang Z, Guo L and Xu G: MiR-758-3p suppresses proliferation, migration and invasion of hepatocellular carcinoma cells via targeting MDM2 and mTOR. Biomed Pharmacother 96: 535-544, 2017.

22. Livak KJ and Schmittgen TD: Analysis of relative gene expression data using real-time quantitative PCR and the $2^{-\Delta \Delta C_{\mathrm{T}}}$ method. Methods 25: 402-408, 2001.

23. Li L, Li B, Zhang H, Bai S, Wang Y, Zhao B and Jonas JB: Lentiviral vector-mediated PAX6 overexpression promotes growth and inhibits apoptosis of human retinoblastoma cells. Invest Ophthalmol Vis Sci 52: 8393-8400, 2011.

24. Bai SW, Li B, Zhang H, Jonas JB, Zhao BW, Shen L and Wang YC: Pax6 regulates proliferation and apoptosis of human retinoblastoma cells. Invest Ophthalmol Vis Sci 52: 4560-4570, 2011.

25. Li X, Yang L, Shuai T, Piao T and Wang R: MiR-433 inhibits retinoblastoma malignancy by suppressing Notch1 and PAX6 expression. Biomed Pharmacother 82: 247-255, 2016.

26. Li Y, Li Y, Liu Y, Xie P, Li F and Li G: PAX6, a novel target of microRNA-7, promotes cellular proliferation and invasion in human colorectal cancer cells. Dig Dis Sci 59: 598-606, 2014.

27. Huang BS, Luo QZ, Han Y, Huang D, Tang QP and Wu LX: MiR-223/PAX6 axis regulates glioblastoma stem cell proliferation and the chemo resistance to TMZ via regulating PI3K/Akt pathway. J Cell Biochem 118: 3452-3461, 2017. 
28. Wang Z, Yao YJ, Zheng F, Guan Z, Zhang L, Dong N and Qin WJ: Mir-138-5p acts as a tumor suppressor by targeting pyruvate dehydrogenase kinase 1 in human retinoblastoma. Eur Rev Med Pharmacol Sci 21: 5624-5629, 2017.

29. Che X, Qian Y and Li D: Suppression of disheveled-axin domain containing 1 (DIXDC1) by MicroRNA-186 inhibits the proliferation and invasion of retinoblastoma cells. J Mol Neurosci 64: 252-261, 2018.

30. Li J, Xu ZW, Wang KH, Wang N, Li DQ and Wang S: Networks of MicroRNAs and genes in retinoblastomas. Asian Pac J Cancer Prev 14: 6631-6636, 2014.

31. Li BR, Xia LQ, Liu J, Liao LL, Zhang Y, Deng M, Zhong HJ, Feng TT, He PP and Ouyang XP: miR-758-5p regulates cholesterol uptake via targeting the CD36 3'UTR. Biochem Biophys Res Commun 494: 384-389, 2017.

32. Ramirez CM, Dávalos A, Goedeke L, Salerno AG, Warrier N, Cirera-Salinas D, Suárez Y and Fernández-Hernando C: MicroRNA-758 regulates cholesterol efflux through posttranscriptional repression of ATP-binding cassette transporter A1. Arterioscler Thromb Vasc Biol 31: 2707-2714, 2011.

33. Meng Y, Zou Q, Liu T, Cai X, Huang Y and Pan J: microRNA-335 inhibits proliferation, cell-cycle progression, colony formation, and invasion via targeting PAX6 in breast cancer cells. Mol Med Rep 11: 379-385, 2015.
34. Georgala PA, Carr CB and Price DJ: The role of Pax6 in forebrain development. Dev Neurobiol 71: 690-709, 2011.

35. Hanson IM: PAX6 and congenital eye malformations. Pediatr Res 54: 791-796, 2003.

36. Xia X, Yin W, Zhang X, Yu X, Wang C, Xu S, Feng W and Yang H: PAX6 overexpression is associated with the poor prognosis of invasive ductal breast cancer. Oncol Lett 10: 1501-1506, 2015.

37. Zong X, Yang H, Yu Y, Zou D, Ling Z, He X and Meng X: Possible role of Pax- 6 in promoting breast cancer cell proliferation and tumorigenesis. BMB Rep 44: 595-600, 2011.

38. Zou Q, Yi W, Huang J, Fu F, Chen G and Zhong D: MicroRNA-375 targets PAX6 and inhibits the viability, migration and invasion of human breast cancer MCF-7 cells. Exp Ther Med 14: 1198-1204, 2017.

39. Wang J, Wang X, Wu G, Hou D and Hu Q: MiR-365b-3p, down-regulated in retinoblastoma, regulates cell cycle progression and apoptosis of human retinoblastoma cells by targeting PAX6. FEBS Lett 587: 1779-1786, 2013.

40. Luo J, Li H and Zhang C: MicroRNA-7 inhibits the malignant phenotypes of nonsmall cell lung cancer in vitro by targeting Pax6. Mol Med Rep 12: 5443-5448, 2015.

41. Zhao Y, Lu G, Ke X, Lu X, Wang X, Li H, Ren M and He S: miR-488 acts as a tumor suppressor gene in gastric cancer. Tumour Biol 37: 8691-8698, 2016. 